\title{
LEPTINA E INSULINA SÉRICA EN ADULTOS CON SOBREPESO Y OBESOS EN RÉGIMEN HIPOCALÓRICO CON ALTO CONTENIDO DE CARBOHIDRATOS COMPLEJOS
}

\section{SERUM LEPTIN AND INSULIN IN OBESE ADULTS ON HYPOCALORIC DIETARY REGIMEN OF HIGH COMPLEX CARBOHYDRATE CONTENT}

\author{
Liseti Solano R., María A., Barón C., Zulay Portillo C., Zuleida Fajardo de B. \\ Universidad de Carabobo. Facultad de Ciencias de la Salud. \\ Instituto de Investigaciones en Nutrición, Venezuela.
}

\begin{abstract}
Leptin acts as a regulatory signal for food intake, body energy balance, body fat content and body weight stability. In order to establish associations among serum leptin, serum insulin and weight loss, 71 obese or overweight adults were assessed by anthropometry and serum determination of leptin at the beginning (B), two weeks (2w) and six weeks (6w) after consuming a dietary regime based on complex carbohydrates intake. Data was analyzed by non parametrical tests for independent and related samples. There was a significant decrease of leptin among $B, 2 w$ and $6 \mathrm{w}$ of similar nature between overweight and obese participants, with all of them reaching normal values at $6 w$ (overweight at $2 w$ and obese at $6 w$ ). Prevalence of normal leptin values $(<15 \mathrm{ng} / \mathrm{mL}$ ) increased among $B, 2 w$ and $6 w$. Leptin levels were significantly higher in obese vs. overweight and in women vs. men with no differences in serum glucose or insulin by sex. There was a significant association between leptin and adiposity indicators (body mass index and abdominal circumference). At $2 w$, but not at $6 w$, a positive correlation between leptin and insulin was found. A dietary regime based on increased consumption of complex carbohydrate (fiber) was efficient to induce favorable changes in anthropometrical and biochemical indicators, especially serum leptin.
\end{abstract}

Key words: leptin; insulin; weight loss; body mass index; fiber.

Este trabajo fue recibido el 18 de Agosto de 2008 y aceptado para ser publicado el 5 de Mayo de 2009.

\section{INTRODUCCIÓN}

Actualmente en los tratamientos de la obesidad, se hace énfasis en el bajo consumo de calorías, lo que favorece la ingesta de carbohidratos complejos con alto contenido en fibra (1). En un régimen dietario, la movilización de la grasa almacenada y su utilización están controlados por la influencia de moduladores positivos como el glucagón y negativos como la insulina, entre otros. Ademas también intervienen los agentes ß-adrenérgicos, la colecistoquinina, el neuropéptido Y (NPY) y la leptina (2-4).

La leptina proviene del gen ob, específico de los adipositos e intimamente ligada a la obesidad, ya que es una señal reguladora negativa sobre la ingesta de alimentos y del balance energético del organismo, para mantener la masa corporal a un nivel constante y además señalar la cantidad de tejido adiposo fino periférico y visceral presente en el cuerpo $(3,5)$.

Las altas concentraciones de leptina sérica en los seres humanos se correlacionan con un elevado contenido de grasa en el cuerpo y con el índice de masa corporal, indicando resistencia a la leptina; lo que sugiere que la mayoría de las personas obesas son insensibles a la producción endógena de la misma (6).

Existen muchas versiones y combinaciones de los alimentos y nutrientes en los regímenes de alimentación a fin de lograr el objetivo de perder peso y control metabólico. Uno de reciente utilización, consiste en reducir las calorías por restricción del aporte de grasas y por aumento del consumo de carbohidratos complejos. 
Se planteó este estudio con el objetivo de evaluar los cambios en los niveles séricos de leptina e insulina, en adultos obesos sometidos a un régimen dietario hipocalórico con alto contenido de carbohidratos complejos, durante un período de 6 semanas a fin de aportar conocimientos sobre la interacción entre las hormonas evaluadas, y corroborar la eficiencia del programa de alimentación utilizado.

\section{SUJETOS Y MÉTODO}

Se trata de un estudio de tipo experimental longitudinal, en adultos con exceso de peso en el cual se midieron los cambios en variables antropométricas, bioquímicas y dietarias entre el inicio, las dos y las seis semanas de una intervención nutricional, a fin de evaluar el impacto de un regimen de alimentación (antes y después).

La población estuvo constituida por adultos con diagnóstico de sobrepeso u obesidad, atendidos en el Centro de Investigaciones en Nutrición de la Facultad de Ciencias de la Salud de la Universidad de Carabobo en Valencia, Venezuela.

La muestra fue intencional, no probabilística dado que los sujetos acudieron voluntariamente ante un anuncio interno para participar en el estudio. Del total de sujetos que concurrieron al llamado $(n=210)$, un $57 \%(n=120)$ aceptó participar y la muestra quedó conformada por un grupo de 71 pacientes, 16 hombres y 55 mujeres (20-55 años), quienes firmaron el consentimiento escrito para participar en el programa especial de tratamiento del sobrepeso y la obesidad, completaron todas las evaluaciones y no presentaron rechazo o manifestaciones gastrointestinales.

Los criterios de inclusión fueron: Ser consumidores frecuentes de cereal en el desayuno, realizar actividad física de ligera a moderada, tener el hábito de comer al menos tres comidas diarias, ser tolerantes a la lactosa, no presentar patologías evidentes, tales como diabetes o hipertensión arterial, no haber estado sometidos a regímenes dietéticos especiales o de pérdida de peso, no fumar por lo menos en los dos meses previos a su participación, no estar recibiendo o haber recibido medicamentos que influyan sobre el apetito o el peso corporal, no haber tenido variaciones de peso mayores a tres kilos en los últimos tres meses.

Se realizaron mediciones antropométricas (peso, talla, circunferencia abdominal e Indice de Masa Corporal) (7), bioquímicas (leptina e insulina sérica por ELISA) y dietarias (tres registros o diarios de consumo dietario, dos en días de semana y uno en fin de semana y un recordatorio de 24 horas) en tres ocasiones, al inicio, a las dos semanas y a las seis semanas de haber iniciado el régimen de alimentación para evaluar los cambios correspondientes. El registro de consumo dietario fue completado por cada sujeto, utilizando juegos de medidas prácticas (tazas y cucharas medidoras) que se entregaron para facilitar la medición. El recordatorio se utilizó para validar los registros de consumo.

Los sujetos fueron clasificados de acuerdo a su índice de masa corporal (IMC), en sobrepeso: IMC 25 a 29,9 $\mathrm{kg} / \mathrm{m} 2$ y obesidad: IMC mayor a $30 \mathrm{~kg} / \mathrm{m} 2$ (8).

Como punto de corte para la leptina, se usó el valor de $15 \mathrm{ng} / \mathrm{mL}$ basado en evidencia previa sobre el rango de concentración de la sustancia en personas con peso normal $(5,9)$.

El esquema del régimen hipocalórico basado en el consumo de un cereal rico en carbohidratos complejos, se cumplió en dos etapas:

- Primeras dos semanas: Sustitución de dos comidas (desayuno y cena o desayuno y almuerzo, según preferencia del participante) por 1 taza de cereal rico en fibra, $1 / 2$ taza de leche descremada y una ración de fruta. Dos meriendas con raciones de frutas y una tercera comida baja en grasa según, la dieta habitual del participante, acompañada de otra ración de fruta.

- Siguientes cuatro semanas: Sustitución de una comida con cereal al día y disminución del consumo de grasas. Se dieron tanto las instrucciones dietarias para promover el consumo de alimentos ricos en carbohidratos complejos como educación nutricional.

Los datos obtenidos de las distintas mediciones realizadas se procesaron con el paquete estadístico SPSS versión 11,0; mediante análisis exploratorio para evaluar la distribución de la muestra y pruebas no paramétricas para comparaciones de medias para muestras independientes o relacionadas (Mann-Whitney; Test de Friedman $\mathrm{Chi}^{2}$ ).

\section{RESULTADOS}

La edad promedio fue 35,94 \pm 9,38 años y el índice de masa corporal promedio fue $32,33 \pm 5,06 \mathrm{~kg} / \mathrm{m}^{2}$, sin diferencias significativas por edad.

En la tabla 1 se observa que al inicio todas las personas calificaron en sobrepeso y obesidad y que a las dos semanas, seis sujetos pasaron de sobrepeso a normalidad y así se mantuvieron hasta el final del estudio. El número de participantes en sobrepeso aumentó a las dos semanas y al final de las seis semanas, indicando que algunos sujetos perdieron peso suficiente para cambiar su diagnóstico de obesidad a sobrepeso, lo cual se observa en la disminución de obesos entre el inicio y el final del período evaluado.

En la tabla 2 se presentan los valores séricos de 
leptina e insulina y del IMC; y su comparación entre los tiempos de evaluación, a fin caracterizar los cambios. Hubo una disminución significativa de los valores circulantes de leptina y del IMC, entre el inicio, las dos semanas y las seis semanas, lo cual fue independiente de si tenían sobrepeso u obesidad.

La distribución de los niveles de leptina en los participantes según la presencia de valores iguales o menores a $15 \mathrm{ng} / \mathrm{mL}$ y mayores de $15 \mathrm{ng} / \mathrm{mL}$ se presenta en la tabla 3. A medida que los participantes mantenían la dieta, se producían cambios en los niveles de leptina que aumentaban la proporción de personas con niveles menores de 15 , es decir, dentro del rango de lo normal.
En la tabla 4 se observan diferencias significativas para la leptina según el sexo de los participantes. Para la insulina sérica no se evidenciaron estas diferencias.

Los resultados presentados en la tabla 5 muestran diferencias significativas dependiente del diagnóstico nutricional para la leptina en los tres tiempos de evaluación, pero no para la insulina.

\section{DISCUSIÓN}

Existen numerosos factores neuroendocrinos responsables de la regulación del metabolismo energético (3), sin embargo, desde el descubrimiento de la leptina, se han realizado numerosas investigaciones con el pro-

\section{TABLA 1}

Distribución de participantes según categoría nutricional y periodo de evaluación.

\begin{tabular}{|c|c|c|c|c|c|c|}
\hline \multirow[b]{3}{*}{ Categorías diagnósticas } & \multicolumn{5}{|c|}{ Periodos de evaluación } & \\
\hline & \multicolumn{2}{|c|}{ Inicio } & \multicolumn{2}{|c|}{ A las dos semanas } & \multicolumn{2}{|c|}{ A las seis semanas } \\
\hline & $\mathbf{n}$ & $\%$ & $\mathbf{n}$ & $\%$ & $\mathbf{n}$ & $\%$ \\
\hline Normales & - & - & 6 & 8,5 & 6 & 8,5 \\
\hline Sobrepeso* & 24 & 33,8 & 22 & 30,98 & 29 & 40,8 \\
\hline Obesidad* & 47 & 66,2 & 43 & 60,56 & 36 & 50,7 \\
\hline
\end{tabular}

\section{TABLA 2}

Estadísticos descriptivos y comparación de variables estudiadas durante el período evaluado.

\begin{tabular}{|c|c|c|c|c|c|c|c|}
\hline Variable & Promedio & D.E. & Mínimo & Máximo & $\begin{array}{l}\text { Rango de } \\
\text { promedio }\end{array}$ & $\begin{array}{c}\text { Test } \\
\text { Chi }^{2}\end{array}$ & $\begin{array}{l}\text { st de Friedman } \\
\text { Significado estadístico }\end{array}$ \\
\hline \multicolumn{8}{|c|}{ LEPTINA (ng/mL) } \\
\hline Inicial & 23,45 & 14,12 & 3,05 & 84,67 & 2,81 & \multirow[t]{3}{*}{104,111} & \multirow[t]{3}{*}{0,000} \\
\hline Dos sem. & 17,46 & 10,52 & 1,89 & 48,51 & 2,08 & & \\
\hline Seis sem. & 11,71 & 7,24 & 1,37 & 30,50 & 1,11 & & \\
\hline \multicolumn{8}{|c|}{ INSULINA $(\mu \mathrm{U} / \mathrm{mL})$} \\
\hline Inicial. & 13,83 & 11,3 & 1,5 & 60 & 2,11 & \multirow[t]{3}{*}{4,45} & \multirow[t]{3}{*}{0,108} \\
\hline Dos sem. & 11.71 & 9.58 & 1,5 & 62,2 & 1,80 & & \\
\hline Seis sem. & 14.57 & 13.08 & 2,5 & 82,5 & 2,10 & & \\
\hline \multicolumn{8}{|c|}{ INDICE MASA CORPORAL $\left(\mathrm{kg} / \mathrm{m}^{2}\right)$} \\
\hline Inicial. & 32,32 & 5,04 & 23,31 & 47,32 & 2,90 & \multirow[t]{3}{*}{93,6} & \multirow[t]{3}{*}{0,000} \\
\hline Dos sem. & 31,62 & 4,93 & 23,9 & 46,20 & 1,79 & & \\
\hline Seis sem. & 31.18 & 4,96 & 23.2 & 44.6 & 1,31 & & \\
\hline
\end{tabular}


pósito de conocer su actividad metabólica y usarla como medida terapéutica contra la obesidad.

En el presente trabajo se encontró una disminución significativa en los niveles circulantes de leptina durante el régimen de alimentación de restricción calórica, basado en el consumo de cereal rico en carbohidratos complejos. Este hallazgo coincide con reportes de disminución de la leptina sérica cuando la ingesta de energía es menor al que necesita el organismo para cumplir sus funciones metabólicas (11-14), y apoyan la idea que la leptina es un regulador psicológico del hambre durante la restricción de energía en seres humanos.

Cuando ocurre pérdida de peso (disminución del tejido adiposo) consecutivamente hay una reducción de la leptina plasmática y un incremento en la producción del Neuropeptido Y por el hipotálamo generando la respuesta biológica al hambre, incrementando el apetito y disminuyendo el gasto de energía. Al contrario, al ganar peso (aumento del tejido adiposo) hay ascenso de la leptina plasmática inhibiéndose la síntesis

\section{TABLA 3}

Distribución de participantes según niveles de leptina y periodo de evaluación.

\begin{tabular}{|c|c|c|c|c|c|c|}
\hline \multirow[b]{3}{*}{ Leptina sérica } & \multicolumn{6}{|c|}{ Periodos de evaluación } \\
\hline & \multicolumn{2}{|c|}{ Inicio } & \multicolumn{2}{|c|}{ Dos semanas } & \multicolumn{2}{|c|}{ Seis semanas } \\
\hline & $\mathbf{n}$ & $\%$ & $\mathbf{n}$ & $\%$ & $\mathbf{n}$ & $\%$ \\
\hline Menor o igual a $15 \mathrm{ng} / \mathrm{mL}$ & 23 & 32,4 & 34 & 47,9 & 49 & 69,0 \\
\hline Mayor de $15 \mathrm{ng} / \mathrm{mL}^{* *}$ & 48 & 67,6 & 37 & 52,1 & 22 & 31,0 \\
\hline
\end{tabular}

TABLA 4

Estadísticos descriptivos y comparaciones de los niveles de leptina e insulina, según género de los participantes en las tres etapas de evaluación.

\begin{tabular}{|c|c|c|c|c|c|}
\hline VARIABLE & GENERO & PROMEDIO & D.E. & $\mathbf{U}$ & $\mathbf{p}$ \\
\hline \multicolumn{6}{|c|}{ LEPTINA (ng/ml) } \\
\hline \multirow[t]{2}{*}{ Inicial } & Masculino & 14,38 & 12,81 & $-3,23$ & 0,001 \\
\hline & Femenino & 25,84 & 13,56 & & \\
\hline \multirow[t]{2}{*}{ Dos semanas } & Masculino & 10.48 & 11,16 & $-3,36$ & 0,001 \\
\hline & Femenino & 19,30 & 9,62 & & \\
\hline \multirow[t]{2}{*}{ Seis semanas } & Masculino & 5.80 & 4,12 & $-4,00$ & 0,000 \\
\hline & Femenino & 13,26 & 7,10 & & \\
\hline \multicolumn{6}{|c|}{ INSULINA $(\boldsymbol{\mu} \mathrm{U} / \mathrm{mL})$} \\
\hline \multirow[t]{2}{*}{ Inicial } & Masculino & 15,44 & 10,25 &,- 972 & 0,331 \\
\hline & Femenino & 13,07 & 11,24 & & \\
\hline \multirow[t]{2}{*}{ Dos semanas } & Masculino & 9.82 & 5,02 &,- 368 & 0,713 \\
\hline & Femenino & 12.28 & 10,03 & & \\
\hline \multirow[t]{2}{*}{ Seis semanas } & Masculino & 12,15 & 6,21 &,- 194 & 0,846 \\
\hline & Femenino & 15,62 & 14,38 & & \\
\hline
\end{tabular}


y secreción del NPY hipotalámico y se observa una disminución en la ingesta de alimentos conjuntamente con un alza en el gasto energético dándose la respuesta a la obesidad (14).

Un estudio de Rissanen P. y col., indica que los valores del leptina sérica por kilogramo de tejido adiposo no cambian perceptiblemente con una modesta pérdida de peso (15). Sin embargo, Weigle el 2003 señaló que cambios pequeños en el peso $(2-4 \mathrm{~kg})$ son suficientes para modificar los niveles de leptina, independiente de sus valores basales (16); lo que coincide con el presente estudio en el cual se observó un cambio de peso tanto en hombres como en mujeres, asociado a los cambios en la concentración sérica de la leptina. Otros autores han encontrado este efecto pero la disminución de los niveles de leptina ha sido mayor debido a la asociación del programa dietario a la práctica de ejercicio o actividad física, incluso en pacientes diabéticos, actividad que en el presente estudio no se promovió (17-20).

La evaluación demostró la estrecha relación de las concentraciones de leptina con los indicadores de adiposidad tales como el índice de masa corporal, el área grasa y otros indices de composición corporal, lo cual ha sido referido previamente (5).

Un aspecto a considerar es la evidencia tan variable de la relación entre la insulina circulante y la leptina. Se conoce que la secreción de leptina inhibe la secreción de insulina por las células $\beta$ pancreáticas, estimula la utilización de la glucosa y estimula la lipólisis $(2,4)$. En el estudio sólo se encontró correlación entre ellas al momento de la segunda evaluación, es decir, a las dos semanas del régimen, relación que no estaba presente al inicio cuando los valores de leptina estaban elevados y al cabo de seis semanas, cuando nuevamente se elevaron.

En este estudio, la disminución de la leptina a las dos semanas se acompañó de disminución de insulina. A las seis semanas, el descenso de la leptina continuó pero la insulina aumentó, incluso a valores mas altos que al inicio. Näslund y colaboradores, han encontrado que puede existir un balance entre estas dos sustancias inducido por los cambios en el peso generados por un régimen dietario, aún cuando el mecanismo implícito no se conoce (21).

El estudio muestra como los cambios en el peso inducidos por un régimen de consumo de carbohidratos complejos modificó la concentración circulante de la leptina. La mayoría de la evidencia previa sobre niveles de leptina en programas de pérdida de peso se ha referido a los cambios inducidos por restricciones calóricas muy

\section{TABLA 5}

\section{Estadísticos descriptivos y comparaciones de de las variables en estudio, según IMC de los participantes en las tres etapas de evaluación.}

\begin{tabular}{|c|c|c|c|c|c|c|c|}
\hline & \multicolumn{3}{|c|}{ Clasificación por IMC } & \multicolumn{2}{|c|}{$\begin{array}{c}\text { Test de } \\
\text { Mann-Whitney }\end{array}$} & \multicolumn{2}{|c|}{$\begin{array}{c}\text { Test de } \\
\text { Kruskal-Wallis }\end{array}$} \\
\hline & Sobrepeso & Obeso & Normal & $\mathbf{U}$ & $\mathbf{p}$ & $\mathrm{Chi}^{2}$ & $\mathbf{p}$ \\
\hline \multicolumn{8}{|c|}{ LEPTINA (ng/ml) } \\
\hline Inicial & $16,44 \pm 9,3$ & $27,24 \pm 15,2$ & - & $-3,13$ & 0,002 & & \\
\hline Dos semanas & $11,73 \pm 7,8$ & $21,13 \pm 10,5$ & $10,45 \pm 6,83$ & & & 11,0 & 0,004 \\
\hline Seis semanas & $9,69 \pm 6,7$ & $14,13 \pm 7,27$ & $4,63 \pm 1,91$ & & & 13,3 & 0,001 \\
\hline \multicolumn{8}{|c|}{ INSULINA $(\boldsymbol{\mu} \mathrm{U} / \mathrm{mL})$} \\
\hline Inicial & $13,11 \pm 12,5$ & $14,08 \pm 10,4$ & - & $-1,08$ & 0,27 & & \\
\hline Dos semanas & $8,31 \pm 4,2$ & $13,28 \pm 11,2$ & $11,03 \pm 6,4$ & & & 2,1 & 0,33 \\
\hline Seis semanas & $13,60 \pm 9,6$ & $15,76 \pm 15,6$ & $13,66 \pm 11,2$ & & & 0,19 & 0,90 \\
\hline \multicolumn{8}{|c|}{ INDICE MASA CORPORAL $\left(\mathrm{kg} / \mathrm{m}^{2}\right)$} \\
\hline Inicial. & $27,23 \pm 1,5$ & $35,37 \pm 4,2$ & - & $-6,85$ & 0,000 & & \\
\hline Dos semanas. & $27,44 \pm 1,6$ & $34,79 \pm 4,0$ & $24,96 \pm 0,7$ & & & 50,7 & 0,000 \\
\hline Seis semanas. & $27,72 \pm 1,6$ & $35,22 \pm 3,8$ & $23,80 \pm 0,6$ & & & 54,8 & 0,000 \\
\hline
\end{tabular}


severas y a predominio de grasas, sin modificaciones en el tipo de carbohidratos utilizados y en algún caso, introduciendo fibra pero no como componente del alimento (21-23). Tal como lo refiere Rosado, faltan estudios semejantes con humanos. Es importante el desarrollo de estudios que asocien los factores ambientales, particularmente la dieta, con la regulación de los niveles séricos y acción de la leptina en humanos (24)

El aporte del presente trabajo es importante al considerar las modificaciones inducidas por un régimen que promociona el consumo de carbohidratos complejos; el cual concuerda con las nuevas recomendaciones para lograr un peso saludable y un menor riesgo de enfermedades crónicas no transmisibles.

\section{RESUMEN}

En situaciones de cambios en el peso, la evidencia sobre la relación entre la leptina y la insulina con el contenido de grasa corporal no es concluyente. Se planteó establecer asociaciones entre leptina e insulina (ELISA) y pérdida de peso (antropometría) durante un régimen hipocalórico alto en fibra, en 71 individuos (sobrepeso/ obesos), al inicio (I), a dos (2s) y a seis semanas (6s) del régimen. Se aplicaron pruebas no paramétricas para muestras independientes y relacionadas. Hubo disminución significativa de la leptina entre I, 2s y $6 \mathrm{~s}$, similar entre sobrepeso y obesidad, normalizandose a las $6 \mathrm{~s}$ (sobrepeso fueron normales a las $2 \mathrm{~s}$ y obesos a las $6 \mathrm{~s})$. Aumentó la prevalencia de valores normales (leptina $<15 \mathrm{ng} / \mathrm{mL}$ ) durante el régimen. La leptina fue significativamente mayor en obesos que en los con sobrepeso y en mujeres que en hombres, sin diferencias significativas por género para la insulina. Asociación significativa entre leptina e indicadores de adiposidad (índice de masa corporal, circunferencia abdominal). A las $2 \mathrm{~s}$, hubo correlación positiva entre leptina e insulina, no evidente a las $6 \mathrm{~s}$. El régimen basado en el consumo de fibra fue eficiente para lograr cambios en los parámetros antropométricos y bioquímicos, en especial, una disminución de la leptina.

Palabras claves: leptina; insulina; pérdida de peso; Indice de Masa Corporal; fibra.

Dirigir la correspondencia a:

Profesora

Liseti Solano R.

Instituto de Investigaciones en Nutrición,

Facultad de Ciencias de la Salud

Universidad de Carabobo, Venezuela

E-mail: 1solano@uc.edu.ve

Agradecimientos: A los sujetos de estudio por su participación y la compañía Kellogg Venezuela por su donación del cereal.

Financiamiento: Consejo de Desarrollo Científico y Humanístico de la Universidad de Carabobo.

\section{BIBLIOGRAFÍA}

1. Sociedad Española para el Estudio de la Obesidad (SEEDO). Consensus for the evaluation of overweight and obesity and the assessment of obesity management. Med Clin 2000; 115: 587-597.

2. Jequier E. Leptin signaling, adiposity, and energy balance. Ann N Y Acad Sci 2002 967:379-88.

3. Simón, E. A. S. Del Barrio. Leptin and obesity. Departamento Nutrición y Bromatología. Facultad de Farmacia. Universidad del País Vasco. 2000.

4. Popovic Vera, Duntas Leonidas H. Brain somatic cross-talk: Ghrelin, leptin and ultimate challengers of obesity. Nutr Neuroscience 2004. 8(1):1-5.

5. Considine RV. Serum Inmunoreactive-leptin concentrations in normal-weight and obese humans. N England J Med 1996; 334 (5):292-5.

6. Scholz GH. Dissociation of Serum Leptin Concentration and Body Fat Content during Long Term Dietary Interventions in Obese Individuals. Horm Metab Res 1996; 28 (12):718-23.

7. Hernández YV. Manual para simplificar la evaluación antropometrica en adultos. Publicaciones Gangazine. 1era Edic. Caracas. 1995.

8. Organización Mundial de la Salud (OMS), 2000 (En línea). Disponible en: http:// www.who.int/ mediacentre/factsheets/fs311/es/index.html). Consultado:03/04/08.

9. Montague CT, Faraooqui S, Whitehead JP, Soos MA, Rau H, Wareham NJ et al. Congenital leptin deficiency is associated with severe early onset obesity in humans. Nature 1997; 387:903-908.

10. Dubuc GR, Phinney SD, Stern JS, Havel PJ. Changes of serum leptin and endocrine and metabolic parameters after 7 days of energy restriction in men and women. Metabolim 1998; 47:429-434.

11. Keim NL, Stern JS., Havel PJ. Relation between circulating leptin concentrations and appetite during a prolonged, moderate energy deficit in women. Am J Clin Nutr 1998, 68(4):761-2.

12. Wisse BE, Campfield LA, Marliss EB, Morais JA, Tenenbaum R, Gougeon R. Effect of prolonged moderate and severe energy restriction and refeeding on plasma leptin concentrations in obese women. Am J Clin Nutr 1999; 70:321-330.

13. Raben A, Astrup A, Vasilaras TH, Prentice AM, Zunft HJ, Formiguera X, Verboeket-van de Venne 
WP, Poppitt SD, Seppelt B, Johnston S, Keogh GF, Saris WH. Frederiksberg C. The CARMEN trial: increased intake of carbohydrates--simple or complex--and unchanged blood lipids in overweight subjects] Ugeskr Laeger 2002; 164(5):627-31

14. Friedman JM, Halaas JL. Leptin and the regulation of body weight in mammals. Nature 1998, 22-395 (6704):763-70.

15. Rissanen P, Makimattila S, Vehmas T, Taavitsainen M, Rissanen A. Effect of weight loss and regional fat distribution on plasma leptin concentration in obese women. Int J Obes Relat Metab Disord 1999. 23(6):645-9.

16. Weigle DS, Cummings DE, Newby PD, Breen PA, Frayo RS, Matthys CC, Callahan HS, Purnell JQ Roles of leptin and ghrelin in the loss of body weight caused by a low fat, high carbohydrate diet. J Clin Endocrinol Metab 2003; 88(4):1577-86.

17. Sartorio A, Agosti F, Resnik M, Lafortuna CL. Effects of a 3-week integrated body weight reduction program on leptin levels and body composition in severe obese subjects. J Endocrinol Invest 2003;26(3):250-6

18. Murakami T, Horigome H, Tanaka K, Nakata Y, Katayama Y, Matsui A. Effects of diet with or without exercise on leptin and anticoagulation proteins levels in obesity. Blood Coagulation \& Fibrinolysis 2007; 18(5):389-394.

19. Monzillo LU, Hamdy O, Horton ES, Ledbury S,
Mullooly C, Jarema C, Porter S, Ovalle K, Moussa A, Mantzoros CS. Effect of lifestyle modification on adipokine levels in obese subjects with insulin resistance. Obes Res 2003; 11(9):1048-54.

20. Tsai AC, Sandretto A, Chung YC. Dieting is more effective in reducing weight but exercise is more effective in reducing fat during the early phase of a weight-reducing program in healthy humans. J Nutr Biochem 2003;14(9):541-9

21. Näslund E, Andersson I, Degerblad M, Kogner P, Kral JG, Rösner S and Hellström PM. Association of leptin, insulin resistance and thyroid function with long-term weight loss.in dieting obese men. J Internal Med 2000; 248:299-308.

22. Sudi KM; Gallistl S; Trobinger M; Weinhandl G; Aigner R; Payerl D; Tafeit E; Moller R; Borkenstein $\mathrm{MH}$. Subcutaneous adipose tissue layers as a stable correlate of leptin in response to short term energy restriction in obese girls. Int J Obes 2001; 25(1S) Issue 5:43

23. Doucet, E.; Imbeault, P.; St-Pierre, S.; Alméras, N.; Mauriège, P.; Richard, D.; Tremblay, A. Appetite after weight loss by energy restriction and a lowfat diet-exercise follow-up. Internat J Obes, 2000; 24(7):906-15

24. Rosado E L, Monteiro J B, Chaia V, Do Lago MF. Efecto de la leptina en el tratamiento de la obesidad e influencia de la dieta en la secreción y acción de la hormona. Nutricion Hospitalaria 2006;21(6):686-693. 